\title{
The calculation of ternary miscibility gaps using the linear contributions method: Problems, benchmark systems and an application to (K, Li, Na)Br
}

\author{
Michel H.G. Jacobs ${ }^{\mathrm{a}, *}$, Harry A.J. Oonk ${ }^{\mathrm{b}}$ \\ a Department of Theoretical Geophysics, Faculty of Earth Sciences, Utrecht University, Budapestlaan 4, NL-3584 CD Utrecht, The Netherlands \\ ${ }^{\mathrm{b}}$ Chemical Thermodynamics Group, Faculty of Chemistry, Utrecht University, Padualaan 8, NL-3584 CD Utrecht, The Netherlands
}

Received 30 August 2005; accepted 17 September 2005

Available online 4 October 2005

\begin{abstract}
Hypothetical systems are useful to enhance the rigor of computerized algorithms and to enhance the applicability of the developed software to physically realistic systems. This paper deals with the calculation of miscibility gaps using the method of the addition of linear contributions. We derived a formalism, which underlies the method, for a multicomponent system. We applied the method to hypothetical miscibility gaps in a single ternary solution form for which the Gibbs energy is characterized by more than two minima. We show that specific Gibbs energy expressions at constant pressure and temperature result in the formation of multiple three-phase fields and multiple critical points. We present an algorithm for the calculation of miscibility gaps in ternary systems, which are characterized by these properties. We applied our method to the system $\mathrm{KBr}-\mathrm{LiBr}-\mathrm{NaBr}$, having the characteristic that the solid form may separate into three phases with the same crystal structure at low temperatures. (C) 2005 Elsevier Ltd. All rights reserved.
\end{abstract}

Keywords: Miscibility gap; Method of linear contributions; Critical points

\section{Introduction}

Phase diagram calculations in our software package XiPT are based on the method of adding linear contributions to Gibbs energy functions. XiPT is a program we use for the construction of a thermodynamic database for materials relevant to geophysics aiming at predicting thermophysical and thermochemical properties at the extreme conditions prevailing in planetary mantles [1-4]. It includes a number of equations of state, for instance the Murnaghan [5], Birch-Murnaghan [6], Morse [7], our own, Vinet et al.'s [8] universal and thermal equations of state based on lattice vibrations, in which the vibrational density of states is described by a Mie-Grüneisen-Debye [9] or Kieffer [10] model. XiPT is the successor of a program called Txy-Calc and in the past, the latter has been applied to systems in which, under specific conditions, miscibility gaps are present, such as $\mathrm{KBr}-\mathrm{LiBr}-\mathrm{NaBr}$ [11] and $\mathrm{Al}-\mathrm{Si}-\mathrm{Zn}$ [12]. The algorithms incorporated in XiPT, based on the method of adding linear

\footnotetext{
* Corresponding author. Tel.: +31 30 2535069; fax: +31 302535030 .

E-mail address: jacobs@geo.uu.nl (M.H.G. Jacobs).
}

contributions, were not published in detail in the open literature, except for basic concepts by Moerkens et al. [13] and in a thesis by Jacobs [14]. The method applied to binary systems has been described extensively by Oonk [15]. In the present work we take the opportunity to briefly outline our method when it is applied to miscibility gaps in a multi-component system. This is done in Section 2. The calculation of miscibility gaps in ternary systems requires the treatment of more than two minima in the Gibbs energy-composition surface. Therefore, we present in Section 3 the application of our method to hypothetical miscibility gaps in a three component system, having this particular characteristic and which we found difficult to calculate in the past, and which we hope may be beneficial for the development of other software codes. In that section we show that the calculation of phase equilibria in a realistic system, $\mathrm{KBr}-\mathrm{LiBr}-\mathrm{NaBr}$, requires the treatment of more than two minima in the Gibbs energy-composition surface.

\section{Application of the method to phase equilibria in a miscibility gap}

The thermodynamic potential for a phase at a specific temperature and pressure in a system with $n$ components is 
given by the well-known expression [16]:

$\mu_{i}=G+\sum_{j=2}^{n}\left(\delta_{i j}-x_{j}\right)\left(\frac{\partial G}{\partial x_{j}}\right)_{x_{k \neq j}}$

where $G$ represents the Gibbs energy of the phase, $x_{j}$ the mole fraction of component $j$, and $\delta_{i j}$ Kronecker's delta $\left(\delta_{i j}=0\right.$ when $i \neq j$ and $\delta_{i j}=1$ when $i=j$ ). As will be shown below it is convenient to write Eq. (1) as:

$\mu_{1}=G-\sum_{j=2}^{n} x_{j}\left(\frac{\partial G}{\partial x_{j}}\right)_{x_{k \neq j}}$

$\mu_{i}=G-\sum_{j=2}^{n} x_{j}\left(\frac{\partial G}{\partial x_{j}}\right)_{x_{k \neq j}}+\left(\frac{\partial G}{\partial x_{i}}\right)_{x_{k \neq i}}$, and $i>1$.

A miscibility gap is characterized by one Gibbs energy function. To avoid confusion we use the expression 'form' to define a mixture with a specific crystallographic structure in the solid state or with no specific structure such as a liquid or a gas. The Gibbs energy and thermodynamic potential in Eqs. (2a) and (2b) refer to a 'form'. At a specific pressure, temperature and overall composition this form may separate into two phases having identical crystallographic structure but different lattice parameters and different compositions. The equilibrium between the two phases is described by the equality of thermodynamic potentials of each component in each phase:

$\mu_{1}\left(x_{2 e}, x_{3 e}, \ldots, x_{n e}\right)=\mu_{1}\left(x_{2 e}^{\prime}, x_{3 e}^{\prime}, \ldots, x_{n e}^{\prime}\right)$

$\mu_{i}\left(x_{2 e}, x_{3 e}, \ldots, x_{n e}\right)=\mu_{i}\left(x_{2 e}^{\prime}, x_{3 e}^{\prime}, \ldots, x_{n e}^{\prime}\right)$, and $i>1$

where $\left(x_{2 e}, x_{3 e}, \ldots, x_{n e}\right)$ and $\left(x_{2 e}^{\prime}, x_{3 e}^{\prime}, \ldots, x_{n e}^{\prime}\right)$ define the equilibrium composition of each phase. Substitution of Eqs. (2a) and (2b) in Eqs. (3a) and (3b) respectively gives:

$$
\begin{aligned}
& G\left(x_{2 e}, \ldots, x_{n e}\right)-\sum_{j=2}^{n} x_{j e}\left(\frac{\partial G}{\partial x_{j}}\right)_{e} \\
& =G\left(x_{2 e}^{\prime}, \ldots, x_{n e}^{\prime}\right)-\sum_{j=2}^{n} x_{j e}^{\prime}\left(\frac{\partial G}{\partial x_{j}}\right)_{e^{\prime}} \\
& G\left(x_{2 e}, \ldots, x_{n e}\right)-\sum_{j=2}^{n} x_{j e}\left(\frac{\partial G}{\partial x_{j}}\right)_{e}+\left(\frac{\partial G}{\partial x_{i}}\right)_{e} \\
& =G\left(x_{2 e}^{\prime}, \ldots, x_{n e}^{\prime}\right)-\sum_{j=2}^{n} x_{j e}^{\prime}\left(\frac{\partial G}{\partial x_{j}}\right)_{e^{\prime}}+\left(\frac{\partial G}{\partial x_{i}}\right)_{e^{\prime}}
\end{aligned}
$$

where the derivatives are taken at the equilibrium compositions denoted by $e=\left(x_{2 e}, x_{3 e}, \ldots, x_{n e}\right)$ and $e^{\prime}=\left(x_{2 e}^{\prime}, x_{3 e}^{\prime}, \ldots, x_{n e}^{\prime}\right)$ respectively. From Eqs. (4a) and (4b) it follows for each component, $i>1$, that:

$$
\left(\frac{\partial G}{\partial x_{i}}\right)_{e}=\left(\frac{\partial G}{\partial x_{i}}\right)_{e^{\prime}} \text {. }
$$

Substitution of Eq. (5) in Eq. (4a) or (4b) gives the relation:

$$
\begin{gathered}
G\left(x_{2 e}, \ldots, x_{n e}\right)-G\left(x_{2 e}^{\prime}, \ldots, x_{n e}^{\prime}\right) \\
+\sum_{j=2}^{n}\left(x_{j e}^{\prime}-x_{j e}\right)\left(\frac{\partial G}{\partial x_{j}}\right)_{e}=0 .
\end{gathered}
$$

In the case that two or more phases are in equilibrium, Eqs. (3a) and (3b) are easily expanded. For $m$ phases taking part in the equilibrium they will lead to:

$$
\begin{aligned}
& \left(\frac{\partial G}{\partial x_{i}}\right)_{e}=\left(\frac{\partial G}{\partial x_{i}}\right)_{e^{\prime}}=\cdots=\left(\frac{\partial G}{\partial x_{i}}\right)_{e^{m-1}}, \\
& \text { and } \quad 1<i \leq n \\
& G\left(x_{2 e}, \ldots, x_{n e}\right)-G\left(x_{2 e}^{\nu}, \ldots, x_{n e}^{\nu}\right)+\sum_{j=2}^{n}\left(x_{j e}^{\nu}-x_{j e}\right)\left(\frac{\partial G}{\partial x_{j}}\right)_{e} \\
& =0, \quad \text { and } \quad 1 \leq v \leq m-1 .
\end{aligned}
$$

Our method uses the property that the addition of a linear contribution term to the Gibbs energy of a form does not affect the equality of thermodynamic potentials of the phases. In other words that the substitution of the Gibbs energy function $G\left(x_{2}, \ldots, x_{n}\right)$ by:

$G^{\prime}\left(x_{2}, \ldots, x_{n}\right)=G\left(x_{2}, \ldots, x_{n}\right)+\sum_{j=2}^{n} l_{j} x_{j}$

where the coefficients $l_{j}$ represent arbitrary constants, does not affect the equalities expressed by Eqs. (5) and (6), or in the more general case by Eqs. (7) and (8), which is easily verified by substitution of Eq. (9) in Eqs. (4a) and (4b).

The key point in our method is to derive the linear contribution coefficients, $l_{j}$. In the equilibrium situation they are given by:

$l_{j}=-\left(\frac{\partial G}{\partial x_{j}}\right)_{e}$.

The linear contribution term, defined by the summation term in Eq. (9), multiplied by minus 1, represents in the case of equilibrium an entity parallel to the common tangent entity. For binary systems this entity is a line, for ternary systems it is a plane etc.

It follows from Eqs. (9) and (10) that the addition of the linear contribution term results in:

$$
\begin{array}{ll}
\left(\frac{\partial G^{\prime}}{\partial x_{j}}\right)_{e}=\left(\frac{\partial G}{\partial x_{j}}\right)_{e}+l_{j}=0 \quad \text { or } \\
\left(\frac{\partial G^{\prime}}{\partial x_{j}}\right)_{e^{\prime}}=\left(\frac{\partial G}{\partial x_{j}}\right)_{e^{\prime}}+l_{j}=0 \quad(\text { for all } j>1) .
\end{array}
$$

Using Eq. (5) it follows for an equilibrium between two phases for all components $i>1$ that:

$$
\left(\frac{\partial G^{\prime}}{\partial x_{i}}\right)_{e}=\left(\frac{\partial G^{\prime}}{\partial x_{i}}\right)_{e^{\prime}}=0 \text {. }
$$

Substituting $G$ by $G^{\prime}$ in Eq. (6) and using Eq. (12) results in:

$G^{\prime}\left(x_{2 e}, \ldots, x_{n e}\right)-G^{\prime}\left(x_{2 e}^{\prime}, \ldots, x_{n e}^{\prime}\right)=0$.

Eq. (12) indicates that the addition of the linear contribution term, given by Eq. (10), has the effect that the common tangent entity to $G^{\prime}$ is horizontal and Eq. (13) shows that the two minima of $G^{\prime}$ are at the same level. In the case where more than two phases are in equilibrium the minima of all these phases are at the same level. The method of linear contributions is based 


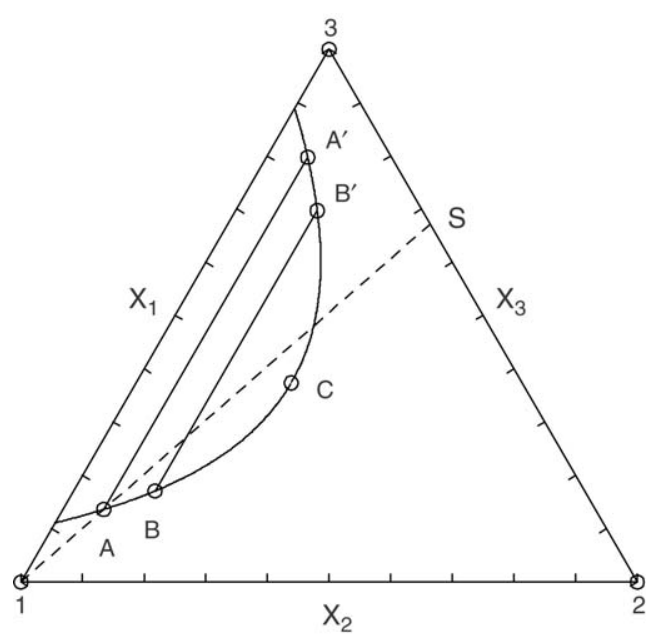

Fig. 1. Miscibility gap in a hypothetical ternary system. The solid lines are tie lines. Point $\mathrm{C}$ is a critical point. The dashed line connecting 1 and $\mathrm{S}$ represents an initial start line.

on finding the linear contribution coefficients, $l_{j}$, such that Eqs. (12) and (13) are satisfied. The next section gives an example to derive these coefficients numerically.

\section{Results and discussion}

In Section 3.1 we demonstrate, at the hand of a model system, an algorithm to derive equilibrium compositions using the method of adding linear contributions. Section 3.2 deals with two hypothetical systems for which the method does not offer a straightforward calculation of equilibrium compositions, and which serve as benchmark systems for our software code. In Section 3.3 we applied the method to a physically realistic system, $\mathrm{KBr}-\mathrm{LiBr}-\mathrm{NaBr}$, having the characteristic that under specific conditions three-phase equilibria are present in the solid form.

\subsection{An algorithm to derive equilibrium compositions}

A simple case to demonstrate the method is a ternary system characterized by a miscibility gap in one of its binary subsystems as depicted in Fig. 1. Our method starts with the definition of a start composition and a start line on which the equilibrium composition of one of the two phases is present. In Fig. 1 these start properties are characterized by component 1 and the line connecting 1 and $\mathrm{S}$ respectively. The composition of the first phase, $\left(x_{2}, x_{3}\right)$, is changed along the line $1-\mathrm{S}$ and in each step the linear contributions are calculated by Eq. (10) resulting in a Gibbs energy $G^{\prime}$. Adding the linear contribution term to $G$ has the effect that, due to Eq. (11), $G^{\prime}$ has a minimum at $\left(x_{2}, x_{3}\right)$. Each step in the composition of the first phase is followed by a search for the second minimum of $G^{\prime}$ belonging to the other phase by changing $x_{2}^{\prime}$ and $x_{3}^{\prime}$. Several minimization methods are available in the literature to accomplish that, e.g. the steepest descent, simplex, generalized gradient method. When the composition of the first phase is between 1 and $\mathrm{A}$, the minimum $G^{\prime}\left(x_{2}^{\prime}, x_{3}^{\prime}\right)$ of the second phase will be above the tangent plane of $G^{\prime}$ at $\left(x_{2}, x_{3}\right)$. Therefore Eq. (13) is clearly

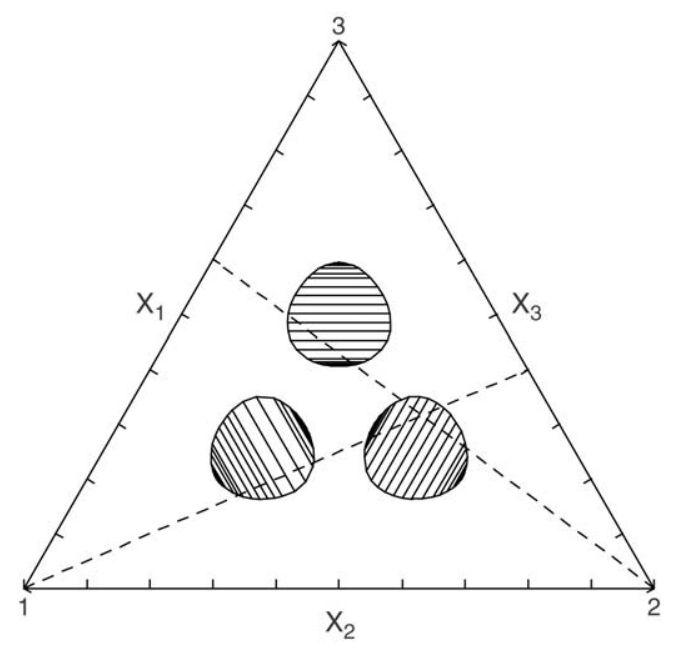

Fig. 2. Miscibility gaps in a hypothetical system calculated with the Gibbs energy given by Eq. (14) and a temperature $T=1450 \mathrm{~K}$. Two dashed initial start lines are given.

not satisfied. When the composition of the first phase increases such that it is between $\mathrm{A}$ and $\mathrm{S}$, the minimum $G^{\prime}\left(x_{2}^{\prime}, x_{3}^{\prime}\right)$ of the second phase will be below the tangent plane of $G^{\prime}$ at $\left(x_{2}, x_{3}\right)$. In that case the composition of the first phase is changed in an iterative procedure until the difference between $G^{\prime}\left(x_{2}, x_{3}\right)$ and $G^{\prime}\left(x_{2}^{\prime}, x_{3}^{\prime}\right)$ is smaller than some criterion value, resulting in tie line $\mathrm{AA}^{\prime}$.

The next tie line, $\mathrm{BB}^{\prime}$, is calculated in a similar way. The difference is that in this case a line parallel to $\mathrm{AA}^{\prime}$ with a user-defined distance to $\mathrm{AA}^{\prime}$ replaces the start line. The start compositions of the two phases are the compositions of $\mathrm{A}$ and $\mathrm{A}^{\prime}$.

In subsequent calculations of tie lines, $x_{2}$ becomes larger than $x_{2}(C)$ and the program finds only one minimum in the Gibbs energy. In that case the program starts from the last calculated tie line and by decreasing the distance between subsequent tie lines point $\mathrm{C}$ is approached as closely as possible within the imposed criteria concerning the accuracy of calculated compositions.

Finally the calculation of tie line $\mathrm{AA}^{\prime}$ is retrieved and the program calculates tie lines until it stops in binary system 1-3.

\subsection{Applying the method to two hypothetical systems}

Generally it is our experience that the method works well but it has a drawback. A weakness of the method is that the initial start line does not always lead to a calculated miscibility gap. For instance, from Fig. 1 it is clear that the initial start line 1-2 or 2-3 does not produce the miscibility gap because no equilibrium composition is on that line. The start line connecting the critical point $\mathrm{C}$ and 2 will not produce the miscibility gap because the program will not be able to find two different minima in the Gibbs energy. Although this weakness is obvious in this simple system, it may lead to problems in other systems. To illustrate that we plotted in Fig. 2 the miscibility gaps of a hypothetical ternary system in which only one form is present. The Gibbs energy of the form is represented by the 


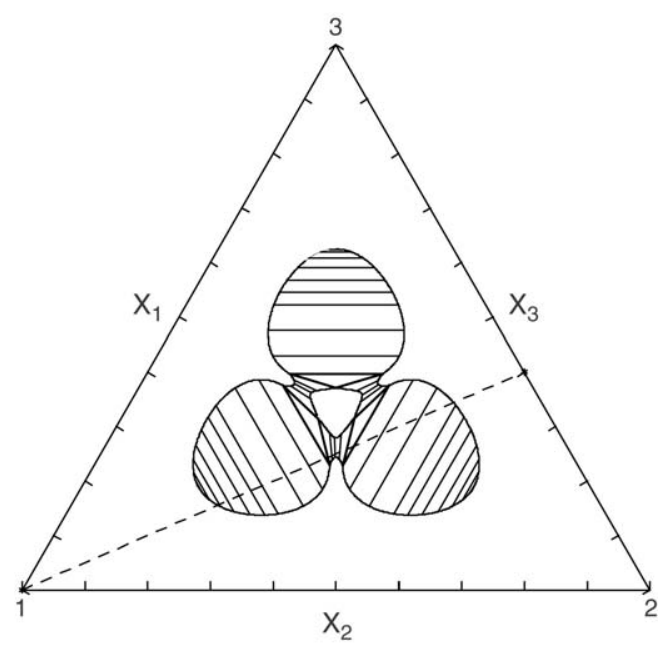

Fig. 3. Miscibility gaps in a hypothetical system calculated with the Gibbs energy given by Eq. (14) and a temperature $T=1410 \mathrm{~K}$. The dashed line indicates a start line.

simple expression:

$G\left(T, x_{2}, x_{3}\right)=R T \sum_{j=1}^{3} x_{j} \ln \left(x_{j}\right)+10^{5} x_{1} x_{2} x_{3}$.

The thermodynamic potentials of the pure substances are omitted in Eq. (14) because that contribution can be considered as the sum of a linear contribution term and a translation constant, and therefore they have no effect on the calculated equilibrium compositions. To make the next points, we have also omitted any excess Gibbs energy in the binary subsystems. It is clear that initial start lines along the binary systems will not produce miscibility gaps. At temperatures higher than $1450 \mathrm{~K}$, the miscibility gaps become smaller and reduce to three single critical points at about $1503 \mathrm{~K}$. At temperatures close to these critical points it is difficult to define initial start lines to produce the three miscibility gaps. Therefore, when a program would be designed which enables the calculation of the three miscibility gaps automatically, by letting the program select initial start lines, a larger computational effort must be invested. Alternatively a program could map the Gibbs energy in composition space and investigate second derivatives with respect to compositions to obtain an estimate on the location of a miscibility gap. That is also accompanied with a larger computational effort.

Another difficulty in the thermodynamic calculation of miscibility gaps in ternary systems is the treatment of three different minima in the Gibbs energy surface. This is illustrated by the cloverleaf shaped miscibility gaps in Fig. 3. In the calculations the complete composition space is considered to find the second and third minimum in the Gibbs energy function $G^{\prime}$. The complete diagram was calculated automatically because all the leaves are connected to one another by three phase equilibria. When a tie line coincides with the edge of a three-phase field, the method produces three minima in $G^{\prime}$, which are at the same level. The calculated three-phase field serves as a point of departure to map two-phase fields, which are connected to it.

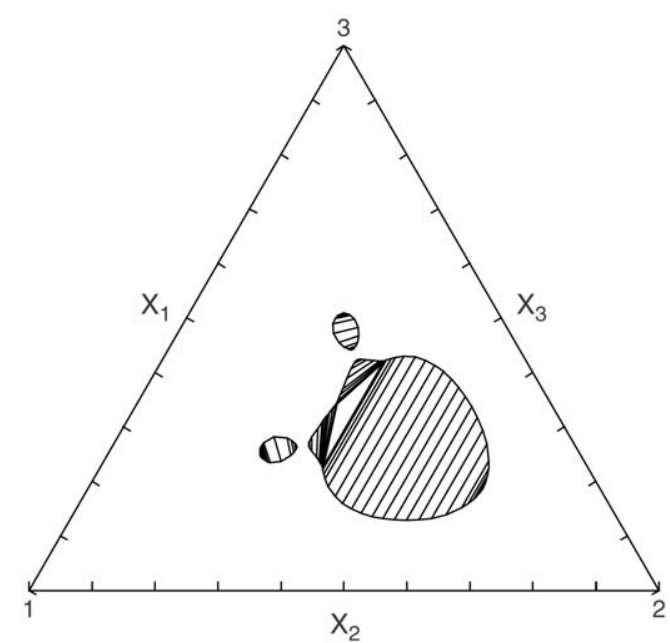

Fig. 4. Miscibility gaps in a hypothetical system calculated with the Gibbs energy given by Eq. (15) and a temperature $T=1520 \mathrm{~K}$.

When temperature is increased to $1412 \mathrm{~K}$ the three main leaves will separate from each other, but after the separation three phase equilibria still exist resulting in nine critical points, three for each leaf. When temperature is further increased to $1433 \mathrm{~K}$, these three phase equilibria disappear and the system contains six critical points, as illustrated in Fig. 2. This process of the change from nine to six critical points is not clearly visible in this system because the involved miscibility gaps are quite small compared to the scale of Fig. 3. The process is better visible when binary system $1-3$ has a positive deviation from ideal mixing behavior as illustrated by the fish shaped miscibility gaps in Fig. 4. The miscibility gaps in Fig. 3, between $1412 \mathrm{~K}$ and $1433 \mathrm{~K}$, show a similar shape to the large shape containing the three phase field in Fig. 4 . To construct Fig. 4 we used the expression:

$G\left(T, x_{2}, x_{3}\right)=R T \sum_{j=1}^{3} x_{j} \ln \left(x_{j}\right)+10^{5} x_{1} x_{2} x_{3}+5000 x_{1} x_{3}$.

Although the systems presented in Figs. 1-4 are hypothetical systems the presence of more than two minima in the Gibbs energy function is important for realistic systems, as we shall demonstrate for $\mathrm{KBr}-\mathrm{LiBr}-\mathrm{NaBr}$.

\subsection{System $\mathrm{KBr}-\mathrm{LiBr}-\mathrm{NaBr}$}

We applied the method of linear contributions to this system in a previous paper [11]. In that work we did not use explicit polynomial functions to include the temperature behaviour of the heat capacity for the pure substances in the solid and liquid phases. We fitted thermodynamic functions extracted from the tables compiled in Barin [17] and Chase et al. [18] and the resulting Gibbs energy expressions for $\mathrm{KBr}, \mathrm{LiBr}$ and $\mathrm{NaBr}$ are listed in Table 1. Following the work of Richet and Fiquet [19], we included a $\ln (T)$ term in the polynomial expression for the heat capacity to better represent the thermodynamic data for $\mathrm{KBr}$. Table 2 and Fig. 5 show that heat capacity values in the 
Table 1

Gibbs energy functions in $\mathrm{J} \mathrm{mol}^{-1}$ at 1 bar pressure of the pure substances $\mathrm{KBr}$, $\mathrm{LiBr}$ and $\mathrm{NaBr}$

$$
\begin{aligned}
G^{\mathrm{sol}}(\mathrm{KBr}, T)= & -409727.343-476.427 \times T+145.445403 \times T \ln (T) \\
& -6.4310431 \times 10^{-2} \times T^{2}-1.158103 \times 10^{-5} \times T^{3} \\
& +2031.055256 \times \ln (T)+39.166186 \times T(-1+\ln (T) \\
& \left.+0.5 \times \ln ^{2}(T)\right) \\
G^{\mathrm{liq}}(\mathrm{KBr}, T)=- & 397300.825+362.386412 \times T-69.873 \times T \ln (T) \\
G^{\mathrm{sol}}(\mathrm{LiBr}, T)= & -351089.981+428.337 \times T-75.045308 \times T \ln (T) \\
& -2.938933 \times 10^{-2} \times T^{2}-1.087973 \times 10^{-5} \times T^{3} \\
& -4291.761094 \times \ln (T) \\
G^{\mathrm{liq}}(\mathrm{LiBr}, T)= & -357684.159+352.539780 \times T-65.27 \times T \ln (T) \\
G^{\mathrm{sol}}(\mathrm{NaBr}, T)= & -381451.842+222.852 \times T-45.986549 \times T \ln (T) \\
& -7.181414 \times 10^{-3} \times T^{2}+90407.389 \times T^{-1} \\
& +1081.854583 \times \ln (T) \\
G^{\mathrm{liq}}(\mathrm{NaBr}, T)= & -357965.4+313.238922 \times T-62.342 \times T \ln (T)
\end{aligned}
$$

The functions were obtained by fitting data compiled in the tables of Barin [17] and Chase et al. [18].

Table 2

Representation of heat capacity compared with values compiled by Barin [17] and Chase et al. [18] for temperatures $\geq 298.15 \mathrm{~K}$

\begin{tabular}{lllll}
\hline $\begin{array}{l}\text { Substance } \\
\text { absolute } \\
\text { deviation in } \\
\text { \% Barin [17] }\end{array}$ & $\begin{array}{l}\text { Average } \\
\text { absolute } \\
\text { deviation in } \\
\text { \% Barin [17] }\end{array}$ & $\begin{array}{l}\text { Maximum } \\
\text { absolute } \\
\text { deviation in \% } \\
\text { Chase et al. [18] }\end{array}$ & $\begin{array}{l}\text { Average absolute } \\
\text { deviation in \% } \\
\text { Chase et al. [18] }\end{array}$ \\
\hline $\mathrm{KBr}$ & 0.26 & 0.09 & 0.31 & 0.12 \\
$\mathrm{LiBr}$ & 0.06 & 0.03 & 0.51 & 0.10 \\
$\mathrm{NaBr}$ & 0.11 & 0.04 & 0.90 & 0.37 \\
\hline
\end{tabular}

compilations [17,18] are represented quite well for $T \geq 298 \mathrm{~K}$. It appeared that only the excess entropy of the liquid phase in the binary system $\mathrm{KBr}-\mathrm{LiBr}$ needed to be re-assessed to obtain a representation of phase diagram data not significantly different from that obtained in our previous work [11]. The reassessed excess entropy is:

$$
\begin{aligned}
S^{\text {Eliq }}(X)= & X(1-X)(-9.51-0.28(1-2 X) \\
& \left.-1.63(1-2 X)^{2}\right) \mathrm{J} \mathrm{K}^{-1} \mathrm{~mol}^{-1}
\end{aligned}
$$

where $X$ denotes the mole fraction of $\mathrm{LiBr}$.

Fig. 3 in Jacobs and Oonk [11] shows that at temperatures smaller than about $520 \mathrm{~K}$ the solid form demixes into three solid phases. Just like for the hypothetical systems described in the previous section, three minima in the Gibbs energy surface of $G^{\prime}$ must be considered. A section at a temperature of $500 \mathrm{~K}$ is given in Fig. 6 showing the locations of these phases in the composition triangle.

The possibility that the Gibbs energy surface has three minima under specific conditions is also present in metallic ternary systems where a solid solution form is not stable in one or two of its binary subsystems. An example is Al-Si-Zn [12], for which the hop form does not exist in the binary systems $\mathrm{Si}-\mathrm{Zn}$ and $\mathrm{Al}-\mathrm{Si}$. To prevent hcp from becoming stable in these two systems the excess Gibbs energy expression has been constructed such that it has high positive values in each binary system. Although the hcp form separates into three hcp phases for temperatures below $739 \mathrm{~K}$, this separation is metastable, because hcp forms stable three-phase equilibria with fcc and

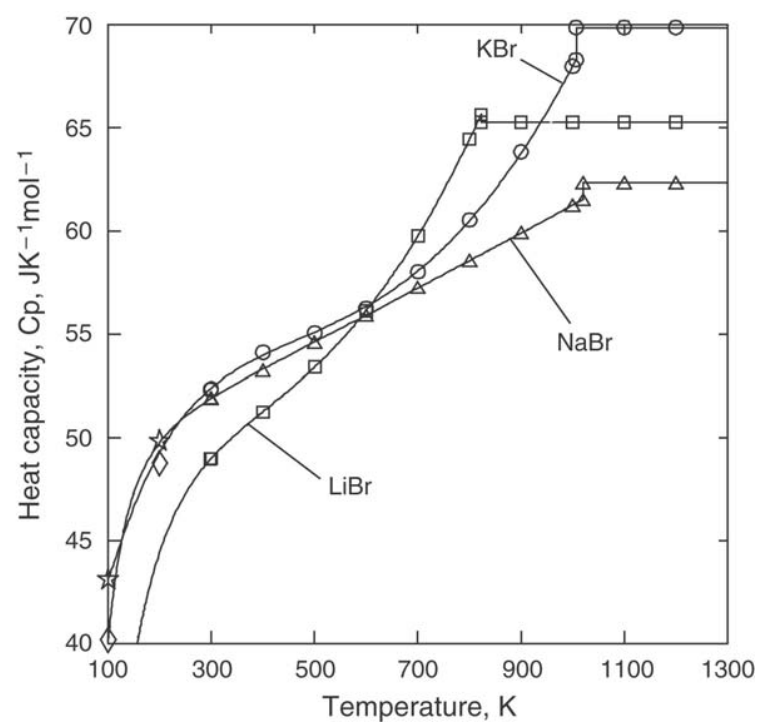

Fig. 5. Heat capacity of the substances $\mathrm{KBr}, \mathrm{LiBr}$ and $\mathrm{NaBr}$ at 1 bar pressure. The circles, triangles and squares indicate values compiled by Barin [17]. Values taken from Chase et al. [18] are stars $(\mathrm{KBr})$ and diamonds ( $\mathrm{NaBr})$.

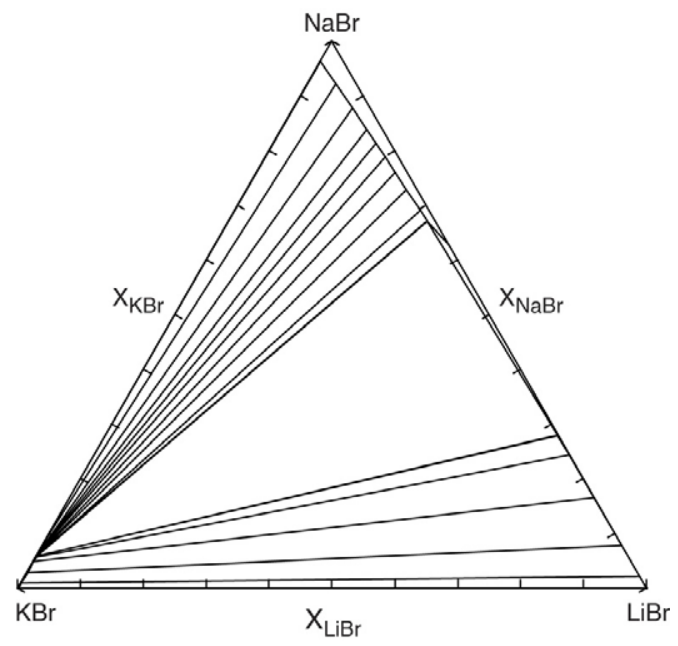

Fig. 6. Isothermal section at $500 \mathrm{~K}$ of the system $\mathrm{KBr}-\mathrm{LiBr}-\mathrm{NaBr}$. Only the solid form is stable.

$\mathrm{Si}$ (diamond) at these conditions. A program, which takes into account only two minima in the Gibbs energy surface of a form, might therefore successfully calculate phase equilibria in $\mathrm{Al}-\mathrm{Si}-\mathrm{Zn}$.

\section{Conclusions}

We derived a formalism, which makes the method of 'the addition of linear contributions' generally applicable to miscibility gaps in multicomponent systems.

We have shown that this method is well suitable to calculate miscibility gaps in ternary systems where one solution form may exhibit three-phase fields. We have presented simple hypothetical test systems having such a characteristic, which may serve as benchmark systems. We have shown that specific miscibility gaps may go undetected by a computational program. We modified our assessment of the 
system $\mathrm{KBr}-\mathrm{LiBr}-\mathrm{NaBr}$ by the introduction of temperature dependent heat capacities of the pure substances and applied the method in the temperature region in which the solid form shows demixing into three solid phases.

\section{References}

[1] M.H.G. Jacobs, H.A.J. Oonk, Phys. Chem. Miner. 28 (2001) 572-585.

[2] M.H.G. Jacobs, H.A.J. Oonk, B.H.W.S. de Jong, Geochim. Cosmochim. Acta 65 (2001) 4231-4242.

[3] M.H.G. Jacobs, B.H.W.S de Jong, Geochim. Cosmochim. Acta 69 (2005) 4361-4375.

[4] M.H.G. Jacobs, A.P. van den Berg, B.H.W.S. de Jong, CALPHAD (2005) (in press).

[5] F.D. Murnaghan, Proc. Natl. Acad. Sci. USA 30 (1944) 244.

[6] F. Birch, J. Geophys. Res 57 (1952) 227-286.

[7] P.M. Morse, Phys. Rev. 34 (1929) 57-64.

[8] P. Vinet, J. Ferrante, J.R. Smith, J.H. Rose, J. Phys. C. 19 (1986)
L467-L473.

[9] O.L. Anderson, Equations of State of Solids for Geophysics and Ceramic Science, Oxford University Press, New York, 1995.

[10] S.W. Kieffer, Rev. Geophys. Space Phys. 17 (1979) 35-59.

[11] M.H.G. Jacobs, R. Jellema, H.A.J. Oonk, CALPHAD 20 (1996) 79-88.

[12] M.H.G. Jacobs, P.J. Spencer, CALPHAD 20 (1996) 307-320.

[13] R. Moerkens, J.A. Bouwstra, H.A.J. Oonk, CALPHAD 7 (1983) 219-269.

[14] M.H.G. Jacobs, The calculation of ternary phase diagrams from binary phase diagrams, Thesis, Utrecht University, 1990.

[15] H.A.J. Oonk, Phase Theory, The Thermodynamics of Heterogeneous Equilibria, Elsevier Scientific Publishing, Amsterdam, Oxford, New York, 1981.

[16] C.H.P. Lupis, Chemical Thermodynamics of Materials, North Holland, 1983.

[17] I. Barin, Thermochemical Data of Pure Substances, Part II, VCH Verlagsgesellschaft mbH, D6940 Weinheim (FRG), 1989.

[18] M.W. Chase Jr., C.A. Davies, J.R. Downey Jr., D.J. Frurip, R.A. McDonald, A.N. Syverud, J. Phys. Chem. Ref. Data (Suppl. 1) (1985).

[19] P. Richet, G. Fiquet, J. Geophys. Res. 96 (1991) 445-456. 